\begin{tabular}{|c|l|}
\hline Title & Mechanism of current leakage through metal $n$-GaN interfaces \\
\hline Author(s) & Oyama, Susumu; Hashizume, Tamotsu; Hasegawa, Hideki \\
\hline Citation & $\begin{array}{l}\text { A pplied Surface Science, 190(1-4), 322-325 } \\
\text { https://doi.org/10.1016/S0169-4332(01)00902-3 }\end{array}$ \\
\hline Issue Date & 2002-05-08 \\
\hline Doc URL & http://hdl.handle.net/2115/5599 \\
\hline Type & article (author version) \\
\hline File Information & A SS190(1-4).pdf \\
\hline
\end{tabular}

Instructions for use 
Applied Surface Science vol. 190, pp.322-325(2002)

\title{
Mechanism of Current Leakage through Metal/n-GaN Interfaces
}

\author{
Susumu Oyama, Tamotsu Hashizume and Hideki Hasegawa \\ Research Center for Integrated Quantum Electronics (RCIQE) and Graduate School of Electronics \\ and Information Engineering, Hokkaido University, Sapporo, 060-8628, Japan \\ Received 10 June 2001
}

\begin{abstract}
Detailed current-voltage-temperature (I-V-T) measurements were performed on the Schottky diodes fabricated on MOVPE-grown n-GaN layers. A large deviation from the thermionic emission (TE) transport was observed in the reverse I-V curves with a large excess leakage. From the calculation based on the thermionic-field emission (TFE) model, it was found that the tunneling plays an important role in the carrier transport across the GaN Schottky barrier even for doping densities as low as $1 \times 10^{17} \mathrm{~cm}^{-3}$. A novel barrier-modified TFE model based on presence of near-surface fixed charges or surface states is proposed to explain the observed large reverse leakage currents.
\end{abstract}

Keywords: GaN, Schottky, leakage current, tunneling, thermionic-field emission, surface trap

\section{Introduction}

$\mathrm{GaN}$ and related compounds have been well established by now not only as materials for blue-emitting photonic devices, but also as materials for high-power/high-frequency field effect transistors (FETs) such as metal-semiconductor FETs (MESFETs) and high electron mobility transistors (HEMTs), power rectifiers and UV photodetectors. Precise and reliable control of the depletion layer width by the Schottky contact without leakage currents is extremely important in these devices. However, GaN-based Schottky interfaces are known to suffer from unusually large leakage currents under reverse bias [1-3]. Excess leakage currents through a Schottky gate strongly affects, not only gate-control and power consumption, but also the noise performance in GaN-based FET devices as recently pointed out [4]. In spite of this, there have been only a few reports on the leakage properties of Schottky contacts in the reverse bias region. The mechanism for large current leakage has not been clarified yet, except that a transport mechanism involving surfaceand/or bulk-trap-assisted tunneling was vaguely suggested without supporting evidence [5,6].

The purpose of this paper is to clarify the mechanism of current leakage through GaN Schottky interfaces by detailed current-voltage-temperature (I-V-T) measurements, especially focusing on the reverse bias characteristics.

\section{Experimental}

Figure 1 shows a schematic illustration of the 
sample structure. Si-doped n-GaN layers $(\mathrm{n}=1.0 \mathrm{x}$ $10^{17} \mathrm{~cm}^{-3}$ ) grown on sapphire substrates by MOVPE were used. A typical value of electron mobility at RT was $500 \mathrm{~cm}^{2} / \mathrm{Vs}$. A ring-shaped ohmic contact was formed on the GaN surface, using a $\mathrm{Ti} / \mathrm{Al} / \mathrm{Ti} / \mathrm{Au}$ $(20 / 80 / 20 / 50 \mathrm{~nm})$ alloy annealed at $600{ }^{\circ} \mathrm{C}$ for $2 \mathrm{~min}$. Schottky contact dots with a diameter of $600 \mu \mathrm{m}$ were formed at the center of the ohmic ring by a conventional vacuum deposition process. $\mathrm{Pt}, \mathrm{Au}$ and Ag were used for Schottky contacts. Just prior to the vacuum deposition, the GaN surfaces were treated in $\mathrm{NH}_{4} \mathrm{OH}$ solution for $15 \mathrm{~min}$ at $50{ }^{\circ} \mathrm{C}$. The detailed XPS analysis revealed that this treatment was very effective in reducing natural oxide components on GaN and AlGaN surfaces [7,8].

$\mathrm{I}-\mathrm{V}$ measurements were done using HP 4156A semiconductor parameter.

\section{Results and discussion}

Figure 2 shows measured forward and reverse $\mathrm{I}-\mathrm{V}-\mathrm{T}$ characteristics of the $\mathrm{Pt} / \mathrm{n}-\mathrm{GaN}$ contact, respectively. From the forward curve at room temperature (RT), an ideality factor, n, of 1.18 and a barrier height, $\phi_{\mathrm{B}(\mathrm{TE})}$, of $0.88 \mathrm{eV}$ were obtained with the thermionic emission (TE) theory. Thus, the I-V behavior apparently follows the TE transport model at RT. However, in the reverse bias region, an excess leakage current flows even at RT, as seen in Fig.2 (b). Furthermore, as a temperature was decreased, deviation from the TE behavior became more and more pronounced in both of the forward and reverse I-V characteristics. To show this clearly, the calculated I-V curves by the pure TE transport

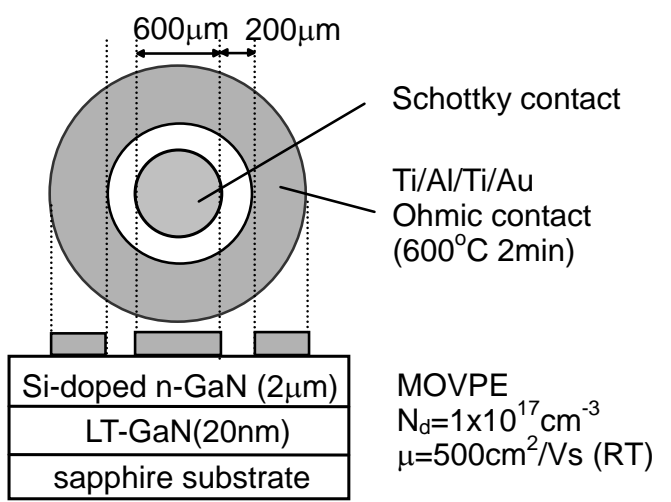

Fig.1 A schematic illustration of the fabricated GaN Schottky structure. assuming $\phi_{\mathrm{B}(\mathrm{TE})}=0.88 \mathrm{eV}$ is plotted by dashed curves in Figs. 2(a) and (b).

In order to explain the observed I-V-T behavior far from the TE mechanism for the Pt/n-GaN Schottky interface, we took account a tunneling transport component based on the thermionic-field emission (TFE) model proposed by Padvani and Stratton [9]. The focus was placed on the reverse bias region at low temperatures where reverse leakage properties showed nearly exponential behavior as expected in the TFE model.

In the TFE model [9], the reverse current transport is due to electrons that are thermally excited from the metal Fermi sea and tunnel through the semiconductor depletion layer to the semiconductor conduction band. In this case, the tunneling probability rapidly increases exponentially with the energy because the excited electrons see thinner and lower barriers, whereas the number of such thermally exited electron decreases exponentially with energy. Thus, TFE takes place at a particular peak energy $E_{m}$ with a narrow Gaussian beam as schematically shown
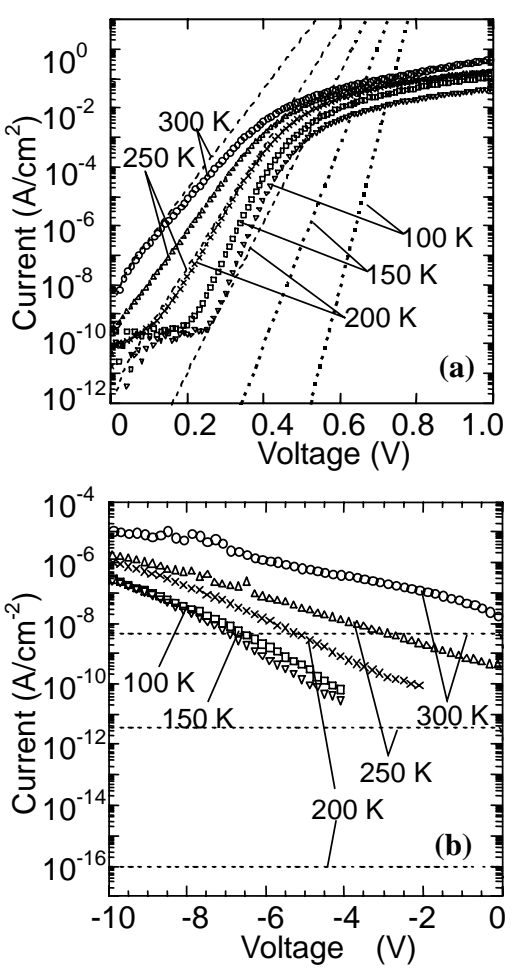

Fig.2 Measured I-V-T curves of the Pt/n-GaN diode in (a) forward bias and (b) reverse bias regions. 
in Fig.3. From such a picture, the reverse current, $J_{R}$, is expressed by the following equations [9].

$$
J_{R}=J_{S} \exp \left[\frac{V_{R}}{k T}-\frac{V_{R}}{E_{00} \operatorname{coth}\left(E_{00} / k T\right)}\right]
$$

with

$$
\begin{aligned}
J_{S}=\frac{A^{*} T \sqrt{\pi E_{00}}}{k} & \sqrt{\left(V_{R}-\phi_{f}\right)+\frac{\phi_{B(T F E)}}{\operatorname{coth}^{2}\left(E_{00} / k T\right)}} \\
& \times \exp \left[-\frac{\phi_{B(T F E)}}{E_{00} \operatorname{coth}\left(E_{00} / k T\right)}\right]
\end{aligned}
$$

where $E_{00}=(q h / 4 \pi)\left(N_{D} / m^{*} \varepsilon_{0} \varepsilon_{s}\right)^{1 / 2}, V_{R}$ is the reverse bias voltage, $k$ is the Boltzmann constant, $\mathrm{T}$ is the absolute temperature, $A^{*}$ is the Richardson constant, $\phi_{\mathrm{f}}$ is $\mathrm{E}_{\mathrm{C}}-\mathrm{E}_{\mathrm{F}}, \phi_{\mathrm{B} \text { (TFE) }}$ is the Schottky barrier height, $\mathrm{h}$ is the Plank constant, $\mathrm{N}_{\mathrm{D}}$ is the donor density, $\mathrm{m}^{*}$ is the effective mass and $\varepsilon_{S}$ is the dielectric constant. $E_{00}$ is the characteristic energy related to the tunneling probability of a triangular potential barrier using the WKB approximation.

For $\mathrm{N}_{\mathrm{D}}=1.0 \times 10^{17} \mathrm{~cm}^{-3}$ in our sample, $\mathrm{E}_{00}$ becomes $3.9 \mathrm{meV}$. A barrier height $\phi_{\mathrm{B}(\mathrm{TFE})}$ of $0.91 \mathrm{eV}$ was obtained by applying the TFE theory to the forward curve at RT and this is very close to $\phi_{\mathrm{B}(\mathrm{TE})}=0.88 \mathrm{eV}$. Using these values and $\mathrm{A}^{*}=26 \mathrm{Acm}^{-2}$ $\mathrm{K}^{-2}$, the reverse I-V-T curves given by Eq.(1) were calculated and the result is plotted by the solid curves in Fig.4 (a) together with the experimental data. The calculation clearly showed nearly exponential dependence of leakage currents on the reverse bias voltage, which was seen in the experiment. However, the measured currents were several orders of magnitude higher than the calculated ones for $\mathrm{T}=250 \mathrm{~K}$ and $300 \mathrm{~K}$. Below $200 \mathrm{~K}$, the discrepancy became over tens orders of magnitude. Then, we tried to fit the experimental

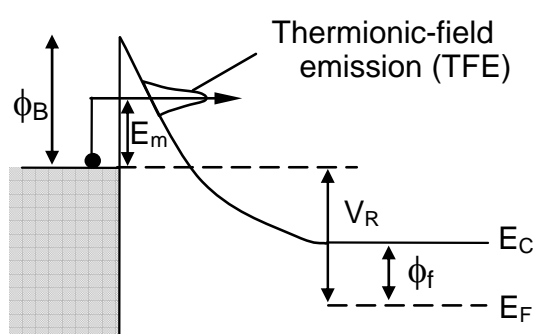

Fig.3 Thermionic-field emission (TFE) model. data using Eq.(1) taking the value of $E_{00}$ as a fitting parameter. An example of reasonably good fit obtained by such an effort is shown in Fig.4 (b). A very good agreement with experiment is seen. However, the fitting value for $\mathrm{E}_{00}$ was $6.0 \mathrm{meV}$, corresponding to the apparent carrier density of $2.3 \mathrm{x}$ $10^{17} \mathrm{~cm}^{-3}$. This is unreasonably too large as compared to the values of $9.0 \times 10^{16}-1.0 \times 10^{17} \mathrm{~cm}^{-3}$ determined by $\mathrm{C}-\mathrm{V}$ measurements in the temperature range from $100 \mathrm{~K}$ to $300 \mathrm{~K}$. These results strongly indicate that a simple TFE model cannot explain the observed large reverse leakage currents quantitatively.

In this respect, several groups vaguely suggested contribution from the trap-assisted tunneling transport for excess leakage currents in the reverse bias region at RT $[5,6]$. Here, presence of discrete deep-level states or a continuum of trap states near the M-S interface is assumed as shown in Fig.5 (a), and they are supposed to provide tunneling paths through the energy barrier after capture of electrons by thermal excitation. However, it is
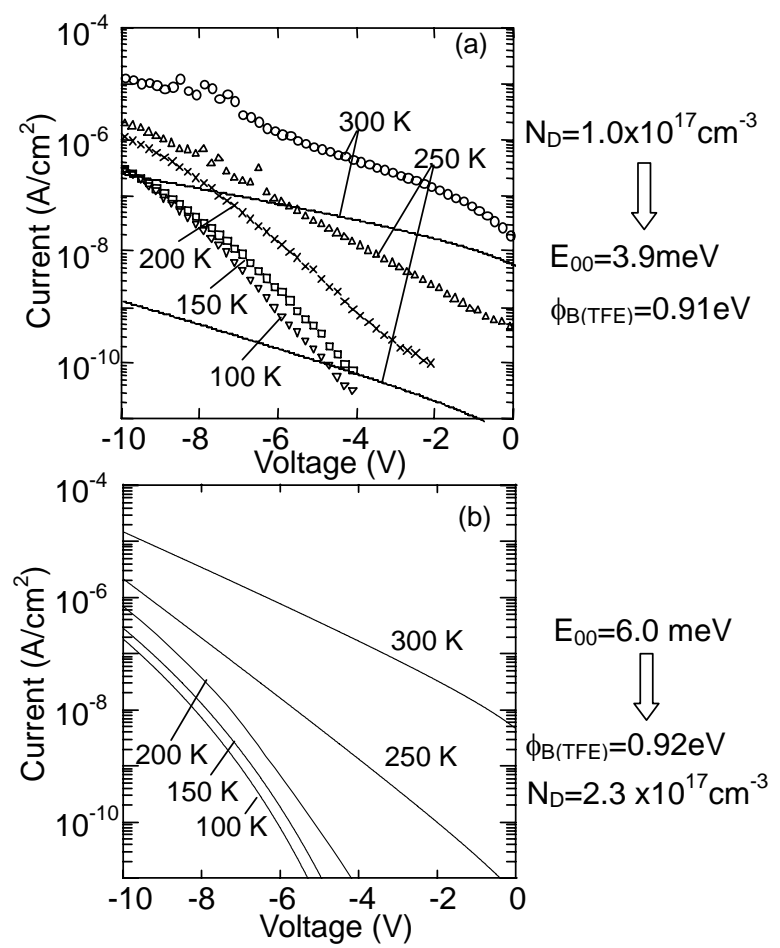

Fig.4 I-V-T curves of $\mathrm{Pt} / \mathrm{n}-\mathrm{GaN}$ in the reverse bias region and calculated I-V-T curves by the TFE model. 
obviously difficult to explain the observed high leakage currents by such a two-step process involving carrier capture process with small capture cross sections. Additionally, thermal excitation process of electrons from metal to the higher trap levels would be suppressed at low temperatures, and the current should be given by Eq. (1) with $\mathrm{E}_{00}=3.9 \mathrm{meV}$ for our sample at low temperatures. Experimentally, however, currents were more than ten orders of magnitude larger. Thus the trap-assisted tunneling model can not explain the experimental data.

Here we propose a barrier-modified TFE model shown in Fig.5 (b). In this model, the potential profile of the Schottky barrier near the M-S interface is modified due to the existence of the high density of positive fixed charge or donor-type deep-surface states. This causes the sharpening of the tunneling barrier profile, leading to enhancement of the probability of the TFE, and giving rise to large reverse leakage. The higher value of $6.0 \mathrm{meV}$ for $\mathrm{E}_{00}$ obtained by fitting can be interpreted as the effective value of $E_{00}$ determining the shape of the tunneling barrier modified by the surface charge near the M-S interface. It is very likely that the present barrier-modified TFE model is applicable to the GaN Schottky interfaces having other metals. In fact, reverse leakage behavior very similar to the results in Figs. 2 and 4 was observed for the $\mathrm{Au}$ and $\mathrm{Ag}$ contacts in our experiments as well as in the results in reported Refs. 2 and 3.

As regards the origin of such states, a recent theoretical calculation [10] has predicted that the nitrogen vacancy $\left(\mathrm{V}_{\mathrm{N}}\right)$ and the oxygen in the nitrogen site $\left(\mathrm{O}_{\mathrm{N}}\right)$ behave as donor states near the conduction band edge. These defects and/or their complex introduced at the GaN surface near the M-S interface seem to be possible candidates for donor-type deep-surface states.

\section{Conclusion}

The temperature dependence of the I-V characteristics of $\mathrm{Pt} / \mathrm{n}-\mathrm{GaN}$ Schottky barriers was measured and analyzed. A large deviation from the thermionic emission transport was observed in the I-V-T behavior with high reverse leakage currents.

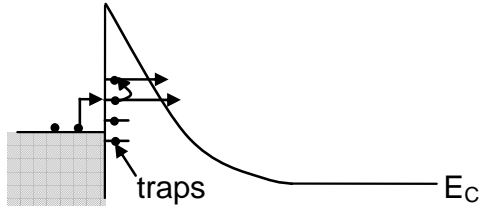

(a) Trap-assisted tunneling

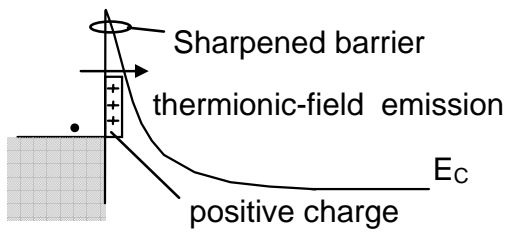

(b) Barrier-modified TFE model

Fig. 5 Models for enhancement of tunneling transport.

The measured reverse I-V-T data can be fitted to the calculation based on the thermionic-field emission (TFE) model. To explain discrepancy between calculated and experimental reverse leakage currents, a new barrier-modified TFE model was proposed.

\section{References}

[1] J.D. Guo, M.S. Feng, R.J. Guo, F.M. Pan, C.Y. Chang, Appl. Phys. Lett., 67 (1995) 2657.

[2] A.C. Schmitz, A.T. Ping, M.A. Kahn, Q. Chen, J.W. Yang,, I. Adesida, J. Electron. Mat., 27(1998) 255.

[3] V. Adivarahan, G. Simin, J.W. Yang, A. Lunev, M.A. Kahn, N. Pala, M. Shur, R. Gaska, Appl. Phys. Lett., 77(2000) 863.

[4] S.L. Rumyantsev, N. Pala, M.S. Shur, R. Gaska, M.E. Levinshtein. M.A. Khan, G. Simin, X. Hu, J. Yang, J. Appl. Phys. 88(2000) 6726.

[5] J.C. Carrano, T. Li, P.A. Grudowski, C.J. Eiting, R.D. Dupuis, J.C. Cambell, Appl. Phys. Lett. 72 (1998) 542.

[6] L. He, X. J. Wang, R. Zhang, J. Vac. Sci. Technol A, 17 (1999) 1217.

[7] T. Hashizume, R. Nakasaki, S. Ootomo, S. Oyama, H. Hasegawa, Mat. Sci. and Eng. B, 80 (2001) 309.

[8] S. Ootomo, S. Oyama, T. Hashizume and H. Hasegawa, IWN-2000 Proc,. IPAP Conf. Ser. 1(2000) 934.

[9] F. A. Padovani, R. Stratton, Solid State Electron. 9(1966) 695.

[10] C.G. Van de Walle, Properties of Gallium Nitride and Related Semiconductors, ed., J.H. Edgar et al., p. 275, INSPEC (1999). 